\title{
A Yoga Intervention for Young Children: Self-Regulation and Emotion Regulation
}

\author{
Roxanne N. Rashedi ${ }^{1} \cdot$ Susan E. Rowe ${ }^{2} \cdot$ Ross A. Thompson ${ }^{3} \cdot$ Emily J. Solari $^{4} \cdot$ Kimberly A. Schonert-Reichl $^{5}$
}

Received: 1 October 2019 / Accepted: 29 May 2021 / Published online: 9 June 2021

(C) The Author(s), under exclusive licence to Springer Science+Business Media, LLC, part of Springer Nature 2021

\begin{abstract}
Yoga-based interventions have been implemented in schools and demonstrated promising results on students' self-regulation outcomes. Nevertheless, there is limited literature on the effects that yoga may have for children in the early primary grades, despite the evidence demonstrating that this is an opportune period in development for early self-regulation. Few studies have focused on young children living in the context of economic difficulty, which can hinder children's development of self-regulatory skills and educational trajectories. The effects of an eight-week yoga intervention on economically disadvantaged pre-kindergarten and kindergarten children's self-regulation and emotion regulation were examined via a paired within-subjects comparison study. Nine classrooms were assigned to the yoga intervention (Treatment First, TxFirst; $n=90$ ) or a wait-list control group (Treatment Second, TxSecond; $n=64$ ). All children were assessed at pre-intervention (Time 1), post-intervention assessment for TxFirst (Time 2), and post-intervention assessment for TxSecond (Time 3). Children demonstrated significant predicted gains on a behavioral task of self-regulation and declines in teacher-rated submissive venting and total behavior problems. Implications for future research are discussed, with a focus on including follow-up assessments and multiple dimensions of fidelity of implementation.
\end{abstract}

Keywords Self-regulation $\cdot$ Emotion regulation $\cdot$ Contemplative practices $\cdot$ Children $\cdot$ Yoga

\section{Highlights}

- Yoga-based interventions have been implemented in schools and demonstrated promising results on students' selfregulation outcomes.

- There is limited literature on the effects that yoga may have for children in the early primary grades, despite the evidence demonstrating that this is an opportune period for early self- regulation.

- Effects of an eight-week yoga intervention on economically disadvantaged pre-kindergarten and kindergarten children's self-regulation and emotion regulation were examined via a paired within- subjects comparison study.

- Children demonstrated significant predicted gains on a behavioral task of self-regulation and declines in teacher-rated submissive venting and total behavior problems.

Self-regulation generally involves monitoring one's thoughts, feelings, and actions and changing them in accordance with the demands of a situation. Successful selfregulation would result in being able to regulate behavior,
Roxanne N. Rashedi

roxanne.rashedi@vanderbilt.edu

Kimberly A. Schonert-Reichl

reichl@uic.edu

1 School of Engineering, Vanderbilt University, 2301 Vanderbilt Place, Nashville, TN 37235, USA
School of Education, University of California, Davis, CA, USA

3 Department of Psychology, University of California, Davis, CA, USA

4 Department of Curriculum Instruction and Special Education, University of Virginia, Charlottesville, VA, USA

5 Department of Psychology, University of Illinois at Chicago, Chicago, IL, United States 
emotion, or attention, especially when the inclination is to act impulsively, in order to achieve a goal or adjust to a situation. With regards to successful kindergarten entry, teachers have ranked self-regulation as more important than content knowledge (Rimm-Kaufman Pianta \& Cox, 2000). Self-regulatory skills have also been identified as robust predictors of learning in school and sustaining relationships (Blair \& Razza, 2007; McClelland et al., 2014). Emotion regulation, a subcategory of self-regulation, involves extrinsic and intrinsic processes responsible for monitoring, evaluating, and modifying emotional reactions (Thompson, 1994). Early deficits in overall self-regulation significantly impede learning (Barkley, 2001). The ability to improve self-regulation calls for investigation, particularly since what constitutes best practices for developing these skills in young children remains unsolidified (Greenberg et al., 2003). Educators have become interested in programs that improve self-regulation during the preschool period, as this is a time characterized by malleability in neurobiological development (Diamond \& Lee, 2011; Diamond, 2013). Preliminary evidence has suggested training self-regulatory skills via various methods, including contemplative practices such as mindfulness and yoga (Davidson et al., 2012).

Contemplative practices strengthen self-regulation by observing when the mind has drifted away from its focus of attention (monitoring) and returning attention to the selected object (cognitive flexibility) (Schmalzl et al., 2014; Shapiro et al., 2006). A regular contemplative practice enhances the ability to sustain engagement of the neural circuits associated with self-regulation (e.g., prefrontal cortex), thus resulting in focused attention and improved emotion regulation (Gard et al., 2014; Zelazo \& Lyons, 2012). On a related note, contemplative practices also develop more bottom-up or more physiologically-based changes, in that bottom-up strategies have been thought of as the regulation of emotion-generative brain areas (e.g., limbic) without recruitiing higher brain areas (e.g., frontal) that are in charge of cognitive forms of regulation, such as reappraisal (Gard et al., 2014).

When children engage in contemplative practices, they cultivate the self-regulatory skills necessary to be aware of their emotions and thoughts (Zelzo \& Lyons, 2012). Such awareness can prompt children to be more capable of responding to others with reflection and perhaps with greater kindness and compassion (Roeser \& Peck, 2009; Zelazo \& Lyons, 2012).

Yoga is one type of contemplative practice and integrates physical postures, breathing exercises, relaxation techniques, and meditation (Gard et al., 2014). In contrast to static meditation, yoga has been shown to be a more developmentally appropriate contemplative practice for young children, in that the postures and breathing exercises are designed to have the child focus on "only one sensation, thought, or emotion at a time"; in turn, the yoga practice creates "manageable, bite- sized" versions of more complicated processes, such as metacognition (p. 103, Moreno, 2017). In yoga, the physical postures are designed to strengthen, as well as stretch and relax the body, and the breathing exercises are integrated into the physical postures to enhance attention to the present (Gard et al., 2014). Yoga is thought to involve a great deal of sensory-motor coordination and may contribute to the neurological foundations of self-regulation (Collins, 2015; Diamond \& Lee, 2011). Furthermore, yoga practices often highlight an embodied form of self-regulation; the practice invites a person to be aware of their thoughts, emotions, and bodily sesations and can cultivate the processes that promote competent self-regulatory skills (Schmalzl et al., 2014).

Moreover, contemplative practices are often embedded in social and emotional learning (SEL) initiatives in schools, partly because these methods focus on improving the awareness of the influence of thoughts and emotions on behaviors, which may contribute to children's abilities to self-regulate, better learn, and relate to others (Lawlor, 2016; Roeser \& Peck, 2009; Semple et al., 2017). Traditional SEL programs, however, tend to start with children's metacognitive skills and then invite children to internalize the feelings (Moreno, 2017). In contrast, contemplative practices start with bodily sensations that prompt children to identify their emotions and then respond to a situation (Moreno, 2017). In this sense, contemplative practices may be more accessible to preschool-age children who are still in the process of developing their metacognitive skills (Moreno, 2017). Equally importantly, contemplative practices connect young children's understanding of emotion in its subjective and expressive features with self-regulatory skills. Consistent with current thinking on the development of emotion regulation, this is likely to enhance the recognition of strategies for managing emotions and provide practice in their implementation (Thompson, 2019).

Several studies involving contemplative practices with elementary-age and preschool-age children have demonstrated promising effects with regards to self-regulation and prosocial behaviors (Flook et al., 2015; Mendelson et al., 2010; Poehlmann-Tynan et al., 2016; Razza et al., 2013; Schonert-Reichl et al., 2015; Schonert-Reichl, 2019; Zelazo et al., 2018). Another team of researchers conducted a randomized controlled trial and investigated the effects that a 12-week yoga intervention had on kindergartener's visual attention, visual-motor precision, and inattentive and hyperactive behaviors (Jarraya et al., 2019). In comparison to the generic physical exercise (PE) and treatment as usual or no physical activities groups, the children in the yoga intervention demonstrated greater improvements in visualmotor precision and attention, as indexed by a direct assessment, and decreases in teacher-rated inattentive and hyperactive behaviors than those in the comparison groups. Moreover, a meta-analysis of 35 school-based 
contemplative practices, including static (that is, not including movement) mindfulness and mindful yoga programs (e.g., programs including static mindful breathing exercises and yoga-based movements), demonstrated a small, positive statistically significant effect on cognitive and socio-emotional outcomes (Maynard et al., 2017). Qualitative studies also suggested that teachers observed the social and emotional benefits their students gain from participating in contemplative practices (Dariotis et al., 2017; Mendelson et al., 2013; Wolff \& Stapp, 2019).

In addition, there has been interest in implementing universal school-based interventions for children who disproportionately face systemic inequities, such that these types of programs, including mindful yoga programs, may help children enhance self-regulation skills (PoehlmannTynan et al., 2016; Razza et al., 2020). This is in part because self-regulation has been identified as a protective factor in previous research and an essential asset for educational attaintment (Blair \& Diamond, 2008; McClelland et al., 2013). Indeed, researchers have examined the effects that an 8-week mindful yoga intervention had on the behavioral and attention regulation of preschoolers facing high levels of trauma in their communities (Razza et al., 2020). Post-intervention results showed significant increases in preschooler's behavioral and attention regulation.

Although there has been progress in this area, there are several significant weaknesses to the current research. First, few yoga and mindfulness interventions have focused on pre-kindergarten and kindergarten-age children, despite the evidence showing that this is a significant period for developing self-regulatory skills (Diamond, 2013). Secondly, few studies have included multiple measures of selfregulation, and follow-up assessments are rarely conducted (Maynard et al., 2017). Thirdly, a growing body of literature has demonstrated that yoga interventions can enhance the self-regulatory skills of young children living in the context of economic difficulty (Poehlmann-Tynan et al., 2016; Razza et al., 2020). Cultivating such self-regulatory skills early in life may be advantageous for children facing economic difficulty, especially when compounded by a year or more of pandemic-related sheltering at home due to the coronavirus pandemic (Hart, 2020; Majumdar et al., 2020). These preliminary findings are promising, but additional investigation in this area is needed. Previous research has tended to prioritize self-regulation as the primary outcome, for example, but yoga practices could become helpful in enabling children to sustain emotional self-control in the context of group learning and enhanced peer interaction, particularly as children transition back to school settings. It is thus informative to also explore the extent to which yoga may help children identify, manage, and cope with difficult emotions, as such emotion regulation skills could be highly relevant to children being able to better navigate everyday adversities. More specifically, yoga may contribute not only to the management of emotional outbursts but also decrease the enlistment of emotion into goal-directed activities (e.g., crying to obtain resources) and enhance alternative problem-solving emotion regulation strategies (Moreno, 2017). Lastly, rarely have prior studies carefully monitored the fidelity of yoga interventions, such as by providing instruction consistently through video materials, especially when teachers are enlisted as instructors because of their ongoing relationships with the children (Feagans Gould et al., 2015).

In light of these findings, we conducted a paired withinsubjects comparison study to assess the effects of training pre-kindergarten and kindergarten teachers to implement a yoga intervention with the support of manualized yoga videos on self-regulation and emotion regulation in children from low socioeconomic status (SES) groups. SES was indexed by the school's eligibility for free or reduced-price lunch programs. To ensure that the effects were attributable to the intervention, teachers were provided with 18 distinct yoga videos. Teachers played the videos and observed and practiced the yoga with children.

This project was a mixed methods study, and we report on the quantitative findings in this article. This study was designed to ask: To what extent did the yoga intervention impact child performance on direct assessments of selfregulation and on teacher ratings of self-regulation and emotion regulation? We hypothesized that the children who received the yoga first (i.e., Treatment First or TxFirst), as well as those who received the yoga second (i.e., Treatment Second or TxSecond), would demonstrate significantly greater self-regulation and emotion regulation on outcome measures after receiving the intervention.

\section{Methods}

\section{Participants}

This study was approved by the Institutional Review Board (IRB) at a research university and adhered to the IRBapproved protocol and consent process. Parental consent was obtained for children's participation. Administrators from three school districts in a Western state that served children who came from low SES groups were recruited. School accountability reports showed that over half of the children in each district received free or reduced-price lunch programs. All pre-kindergarten and kindergarten teachers who had taught at least a year at the six schools were invited to participate $(n=9)$. Teachers received a $\$ 15$ gift card at each time point for completing the surveys, a set of new yoga mats for their class, a breathing ball, a chime, and a yoga mat for their own practice. 
Teachers had a similar amount of classroom teaching experience, with the average being 15.6 years for those in TxFirst and 15.25 for teachers in TxSecond. Prior to participating in the study, three teachers reported that they had no exposure to yoga, whereas the remaining teachers had personally practiced yoga at home or a studio and/or occasionally incorporated yoga into their classrooms by using free resources on YouTube, such as Cosmic Kids Yoga and GoNoodle.

Initial recruitment consisted of 166 children. There were seven children from TxFirst and five children from TxSecond who moved away from the area before the intervention started. There were no other drop-outs. The final participation rate was $93 \%$. The final sample size consisted of 154 children aged 4 to 6 years $(M=65$ months, $S D=5.4$ months; $51 \%$ males) from nine classrooms (see Table 1). As shown in Table 1, and consistent with the sociodemographic composition of the sample, children were predominantly WhiteHispanic as well as White-nonHispanic, and their parents consisted primarily of high school graduates or those with some college education beyond the diploma.

Table 1 Child and parent demographics

\begin{tabular}{|c|c|c|c|c|}
\hline \multirow[t]{3}{*}{ Characteristic } & \multirow{2}{*}{\multicolumn{2}{|c|}{$\frac{\text { TxFirst }}{(n=90)}$}} & \multirow{2}{*}{\multicolumn{2}{|c|}{$\frac{\text { TxSecond }}{(n=64)}$}} \\
\hline & & & & \\
\hline & Count & $\%$ & Count & $\%$ \\
\hline \multicolumn{5}{|l|}{ Grade } \\
\hline Kindergarten & 34 & $37.8 \%$ & 28 & $43.8 \%$ \\
\hline Pre-Kindergarten & 56 & $62.2 \%$ & 36 & $56.3 \%$ \\
\hline \multicolumn{5}{|l|}{ Gender } \\
\hline Male & 48 & $53.3 \%$ & 32 & $50.0 \%$ \\
\hline Female & 42 & $46.7 \%$ & 32 & $50.0 \%$ \\
\hline \multicolumn{5}{|l|}{ Race } \\
\hline White-Non Hispanic & 27 & $30.0 \%$ & 17 & $26.6 \%$ \\
\hline White-Hispanic & 43 & $47.8 \%$ & 24 & $37.5 \%$ \\
\hline African-American & 3 & $3.3 \%$ & 1 & $1.6 \%$ \\
\hline $\begin{array}{l}\text { Native Hawaiian or Pacific } \\
\text { Islander }\end{array}$ & 3 & $3.3 \%$ & 1 & $1.6 \%$ \\
\hline Asian & 7 & $7.8 \%$ & 10 & $15.6 \%$ \\
\hline American-Indian/Alaskan Native & 1 & $1.1 \%$ & 0 & $0.0 \%$ \\
\hline Multiple Races & 4 & $4.4 \%$ & 10 & $15.6 \%$ \\
\hline Unreported & 2 & $2.2 \%$ & 1 & $1.6 \%$ \\
\hline \multicolumn{5}{|l|}{ Parent Education } \\
\hline Some K-12 & 4 & $4.4 \%$ & 3 & $4.7 \%$ \\
\hline High school graduate & 24 & $26.7 \%$ & 22 & $34.4 \%$ \\
\hline Some college, no degree & 21 & $23.3 \%$ & 23 & $35.9 \%$ \\
\hline Associate's degree, occupational & 8 & $8.9 \%$ & 8 & $12.5 \%$ \\
\hline Associate's degree, academic & 5 & $5.6 \%$ & 4 & $6.3 \%$ \\
\hline Bachelor's degree & 15 & $16.7 \%$ & 2 & $3.1 \%$ \\
\hline Graduate degree & 13 & $14.4 \%$ & 2 & $3.1 \%$ \\
\hline
\end{tabular}

\section{Procedures}

\section{Components of the yoga intervention}

The intervention included yoga-based postures, songs, breath regulation, and relaxation exercises conveyed in 18 videotaped lessons lasting approximately $10 \mathrm{~min}$ apiece. Each lesson included the following components: (a) an attention practice that encouraged focusing on the body and to the present; (b) yoga postures combined with breathing techniques aimed at developing breath awareness and regulation and awareness of sensations and emotions; and (c) a guided relaxation (See Appendix). Animal-like yoga postures, such as cobra, monkey, downward dog, and nature postures, such as mountain, tree, and rock, were common postures, as children often enjoy these poses.

Teachers were instructed to play the video and practice the yoga along with the students, while also monitoring student engagement. Teachers were provided with six videos weekly, and the lessons included postures and sequences from the prior week as well as introducing new postures. Teachers played the weekly videos in any order for each given week of the eight-week intervention. Lessons were repeated to reinforce the skills that the students learned the previous week and to address teachers' comments during the yoga teacher training on simplifying the sequences.

\section{Yoga dosage}

Teachers were instructed to teach the lessons six times weekly with opportune times for practice, such as before circle time (i.e., a time when the children sit in a circle and participate in an activity), or after lunch, for eight weeks. The goal was for teachers to integrate yoga sessions into their classroom schedule, such that the yoga practice functioned as a transition to the next activity. Five teachers implemented the yoga three times weekly, twice per day. The remaining four teachers implemented the yoga five times weekly and one day the yoga was twice. The total amount of yoga each teacher implemented in their class was approximately $8 \mathrm{~h}$ over the eight-week intervention period. Teachers in the TxSecond group did not have access to the yoga videos until their intervention started.

\section{Yoga teacher training}

All teachers participated in $10 \mathrm{~h}$ of yoga training led by the research team at the same time. Teachers in TxSecond were instructed to not apply any of the yoga-based practices in their instruction until their intervention time started. Trainings were held primarily after school and took place four months prior to implementation. Each session involved breathing exercises, practicing and discussing the lessons 
and yoga videos, and a guided relaxation. Each training session included time for teachers to practice the same lessons that their children would receive and discuss potential barriers to implementation, as well as learn how to create a constructive environment for a yoga practice (e.g., how to provide assistance to children). Two videos were piloted during the fall semester prior to the intervention, and teachers' comments regarding the camera angle and sound were incorporated into the development of the videos.

\section{Treatment allocation}

Prior to the intervention, stratified random assignment was used to randomly assign classrooms to receiving the yoga first (i.e., TxFirst), or receiving the yoga second (i.e., TxSecond) (Hedges \& Rhoads, 2010; Stuart \& Rubin, 2008). Grade level was used to match classrooms. This variable was controlled when randomizing the classrooms to the yoga or wait-list control condition. The objective was to evenly divide the two classrooms (i.e., pre-kindergarten and kindergarten) and match by grade and school when possible (see Fig. 1). In short, TxFirst: 1 school had 2 classes and 3 schools had 1 class; TxSecond: 1 school had 2 classes, 2 schools had 1 class.

Fig. 1 Study stratification

\section{Data collection}

Time 1 refers to the pre-intervention stage when no children had received yoga treatment. Time 2 refers to the administration of the assessments one week following the first eight-week yoga intervention for children in TxFirst. Children in the TxSecond group did not receive any yoga treatment. Time 3 refers to an eight-week follow-up assessment for the children in TxFirst (who did not continue the yoga beyond Time 2) and an administration of the assessments one week following the first eight-week yoga intervention for children in TxSecond. At Time 1, Time 2, and Time 3, teacher reports of children's self-regulation and emotion regulation were collected, and research assistants administered direct assessments measuring self-regulation. Assessments were video recorded. Research assistants and teachers were not blinded to group allocation.

\section{Measures}

\section{Self-regulation}

The self-regulation measures were: (a) the Toy Wrapping, Toy Waiting, and Pencil Tapping Tasks from The

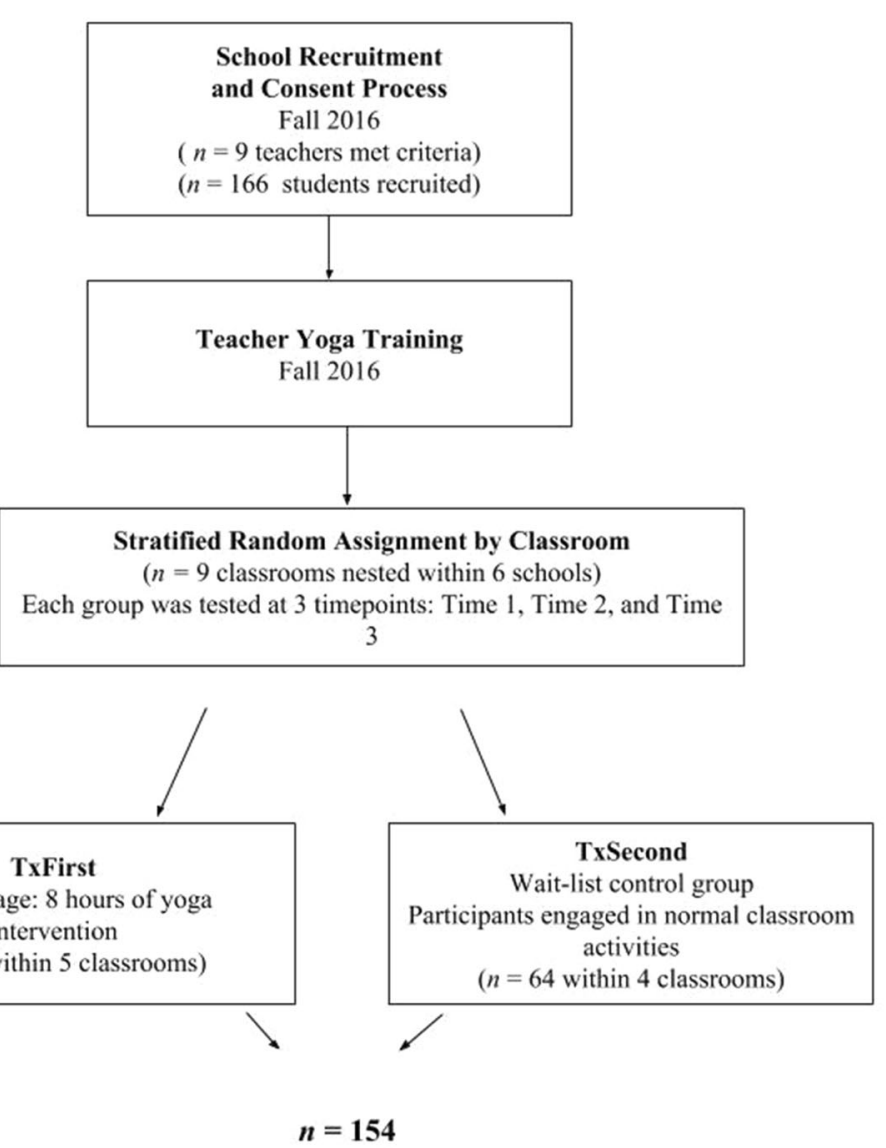


Preschool Self-Regulation Assessment (PSRA; SmithDonald et al., 2007); (b) The Head Toes Knees Shoulders Task (HTKS; Ponitz et al., 2009); and (c) teacher ratings on the Strengths and Difficulties Questionnaire (SDQ; Bourdon et al., 2005; Von Suchodoletz et al., 2009).

The PSRA is an observer-rated behavioral battery assessing self-regulation, especially inhibitory control and cognitive flexibility (Smith-Donald et al. 2007). In the Toy Wrapping task, the child was informed that a surprise was waiting for them, but the researcher needed to first wrap it. The child was positioned away from the research assistant who sat in a chair at a $90^{\circ}$ angle facing away from the child's table. The child was asked not to peek while the researcher wrapped the toy. After $60 \mathrm{~s}$, the researcher presented the child with the wrapped toy. The child's latency to peek (number of seconds before peeking within the 60 second interval) was recorded. In the Toy Wait task, the researcher presented the child with the wrapped toy from the Toy Wrap task and instructed the child to not touch it. The researcher pretended to be busy tidying up and recorded the child's latency to touch the wrapped toy (number of seconds before touching within a $60 \mathrm{~s}$ interval). The child was then invited to unwrap the toy. In the pencil-tapping task, the researcher presented the child with a pencil and introduced two rules: (1) tap two times on the table when the researcher taps once and (2) tap one time on the table when the researcher taps two times. After four practice trials, the researcher administered 16 trials (eight one-tap and eight two-tap trials). The total number of correct responses was divided by the 16 trials. The PSRA has shown high internal consistency ( $\alpha$ $=0.87$ ) and confirmed its measurement equivalence across diverse groups (Smith-Donald et al. 2007).

The HTKS Task was also used to measure self-regulation, especially inhibitory control (Ponitz et al., 2009). The HTKS task required the child to do the opposite of what the researcher instructed, such that the child had to inhibit the dominant response of imitating the researcher, as well as remember and focus on the rules of the task to achieve the goal. For example, when the researcher said, "Touch your head," the child had to remember to touch their toes. There were three practice trials, each with 4 practice items, and three testing portions, with a total of 30 test trials. Computing the scores for each test trial included the following: the child received a 0 (incorrect), 2 (correct), or 1 (selfcorrect) score for each trial, with the total correct responses ranging from $0-60$.

In addition to the direct assessments, self-regulation was also measured by teacher ratings on the SDQ, which emphasizes behavioral indications of inhibitory control and emotion self-regulation (Bourdon et al., 2005; von Suchodoletz et al., 2009). Teachers were asked questions about the target child's behavior in the past six months and responses were based on a 3 -point scale $(0=$ not true $; 1=$ somewhat true $; 2$ = certainly true). We used the hyperacivity/inattention subscale (5 items) and total behavioral difficulties (20 items). Each subscale has adequate teacher test-retest reliability $(0.85$ and 0.84$)$ and adequate teacher internal consistency reliability $(\alpha=0.83$ and $\alpha=0.82$; Stone et al. 2010). The SDQ has a reporting period of six months, but our expectation was that teacher responses would be significantly influenced by the intervention, and the results suggest that this was true. In the current sample, teacher internal consistency reliability was evaluated at each time point. For hyperactivity/ inattention, internal consistency reliability for Time 1 , Time 2, and Time 3 was $\alpha=0.89, \alpha=0.89$, and $\alpha=0.90$, respectively. For total behavioral difficulties, internal consistency reliability for Time 1 , Time 2 , and Time 3 was $\alpha=0.87, \alpha=0.88$, and $\alpha=0.87$, respectively. The internal consistency reliability for the hyperactivity/ inattention subscale was within the range reported by Stone et al. (2010), and in the case of total behavioral difficulties, we found our reliability coefficients higher than the range of reported reliability.

\section{Emotion regulation}

The Children's Emotion Regulation Processes Questionnaire (ERQ) was also used to measure emotion regulation (Meyer et al., 2014). The ERQ was based on Bernzweig, Eisenberg, and Fabes's study (Bernzweig et al., 1993), and adapted by Meyer et al. (2014) for teachers. Teachers were presented with eight scenarios about daily conflicts focused on the emotions of sadness and anger and a list of strategies. Teachers rated the likelihood that the target child would engage in each strategy on a 7-point Likert scale ( 1 = extremely untrue of your student; $2=$ quite untrue of your student; 3 = slightly untrue of your student; $4=$ neither true nor false of your student; $5=$ slightly true of your student; $6=$ quite true of your student; $7=$ extremely true of your student). Strategies were factor-analyzed into four groups: (a) attention-focusing (e.g., think about positive things); (b) dominant venting (e.g., hit or yell to obtain goal); (c) submissive venting (e.g., cry to obtain goal); and (d) problem-emotion focusing (e.g., approach an adult to seek emotional support). Higher scores on attention-focusing indicated that when the target child faced daily conflicts the child would think about something different, play a new game, or talk about something else, which is a benefit to competent emotion regulation. Higher scores on problem-emotion focusing indicated that the target child would approach another child or adult for help or to intervene in daily conflicts. Lower scores on submissive venting and dominant venting indicated that the target child cried, hit, or yelled significantly less to navigate daily 
conflicts, which contributes to emotion coping. The ERQ has adequate internal consistency reliability: attention focusing, $\alpha=0.78$, dominant venting, $\alpha=0.93$, submissive venting, $\alpha=0.81$, problem-emotion focusing, $\alpha=0.70$ (Meyer et al., 2014). In the current sample, there was strong internal consistency reliability for each subscale for the ERQ at each time point. At Time 1, attention focusing $\alpha=$ 0.99 , dominant venting $\alpha=0.98$, submissive venting $\alpha=$ 0.98 , and problem-emotion focusing $\alpha=0.93$. At Time 2 , attention focusing $\alpha=0.98$, dominant venting $\alpha=0.98$, submissive venting $\alpha=0.98$, and problem-emotion focusing $\alpha=0.91$. At Time 3, attention focusing $\alpha=0.98$, dominant venting $\alpha=0.96$, submissive venting $\alpha=0.96$, and problem-emotion focusing $\alpha=0.91$.

In addition, teacher ratings on the SDQ for emotion regulation (5 items) were also collected. This subscale has adequate teacher test-retest reliability (0.72) and adequate teacher internal consistency reliability $(\alpha=0.73)$ (Stone et al., 2010). Within our own sample, internal consistency reliability for the emotion regulation SDQ subscale for Time 1, Time 2, and Time 3 was $\alpha=0.84, \alpha=0.74$, and $\alpha$ $=0.70$, respectively, within the range of reported reliabilities for this subscore in Stone et al. (2010).

\section{Parent and child demographic questionnaire}

Parents completed a demographic questionnaire with information about themselves and their child including age, sex, race, ethnicity, medical history, and education. This form was completed at Time 1 .

\section{Fidelity of implementation (FOI)}

FOI was assessed through checklists that teachers submitted weekly. After every session, teachers rated the degree to which they completed segments of the video on a scale ranging from 1 to 3 , with 1 being "all," 2, "some," and 3 as "none." For example, if teachers skipped the conclusion of the lesson, in which children were guided through a brief mindful listening activity (e.g., listening to the sound of the chime and then coming up to seated when they could no longer hear the sound of the chime), then the teacher rated that segment of the video as "none" or a 3. The average number of yoga sessions a teacher implemented during the eight-week period was 41 , with the FOI being $87 \%$ on average.

\section{Measures of attendance}

To assess the attendance of the intervention, drop-out rates were monitored, in addition to the number of yoga sessions the average child in the intervention attended. The average child in the intervention attended 43.6 of 48 sessions of yoga $(S D=4.38)$. According to teachers, the primary reason for non-attendance was absence due to child illness.

\section{Coding Procedure for Tasks}

\section{Toy task inter-rater reliability}

To determine inter-rater reliability for the Toy Wrapping and Toy Waiting tasks, thirty percent $(n=43)$ of students' video recorded assessments were randomly selected for each time point (Time 1, Time 2, and Time 3) and were rated by two coders. This approach was used as an acceptable alternative to a fully crossed study design as having enough raters for a fully crossed study can be time intensive and costly (Hallgren, 2012). Coders were trained to reliability criteria of 0.80 or greater on tasks. Inter-rater reliability was $88.2 \%$ agreement between raters for both the Toy Wrap and Toy Waiting tasks. Cohen's kappa coefficients for the Toy Wrapping tasks and Toy Waiting tasks were 0.629 and 0.677 , respectively, indicating susbstantial agreement (Cohen, 1988). Inter-rater reliability and Cohen's kappa were utilized to provide two measures of rater reliability rather than relying only on one (McHugh, 2012).

\section{Data Analyses}

\section{Group Equivalence}

Following stratified random assignment by classroom, there were no significant age differences between groups (TxFirst, $\mathrm{M}=66$ months, TxSecond, $\mathrm{M}=65$ months, $t$ $(152)=1.26, p=0.262)$. Independent samples $t$-tests were run to examine differences in the demographic variables; there were no significant differences between TxFirst and TxSecond demographics concerning grade, $t(152)=0.741$, $p=0.460$, gender, $t(152)=0.406, p=0.686$, and race. For race, we examined whether there was a difference in proportions of White-non-Hispanic and White-Hispanic (the largest racial/ethnic subpopulations in our study) and found no significant difference between TxFirst and TxSecond, $t$ $(152)=-0.463, p=0.644$, and $t(152)=-1.266, p=$ 0.207 , respectively. We examined parent education levels as a binary variable, which was whether the parent with the highest education level had a Bachelor's degree or higher and found a significant difference between TxFirst and TxSecond, $t(152)=-3.905, p<0.001$. TxFirst parents were more likely to have obtained a Bachelor's degree or higher and we included this predictor in our initial models, but it was found to be not a significant predictor of our outcome variables. Consequently, it was dropped from further analysis. Additional $t$-tests were conducted to check for preexisting differences between groups at Time 1 on the 
outcome measures. At Time 1, TxSecond had significantly higher total behavioral difficulties and emotion regulation skills on the SDQ than TxFirst, $t(152)=1.98 p=.049$ and $t$ $(152)=2.99, p=0.003$. At Time 1 , TxFirst had significantly lower dominant venting and submissive venting scores on the ERQ compared to TxSecond, $t(152)=2.23, p$ $=0.027$ and $t(152)=2.73, p=0.007$.

The Shapiro-Wilk Test was used to check normality for all measures. Scales that violated normality were transformed, with the exception of the Pencil Tapping task, the conduct subscale, and the Toy Wait and Toy Touch tasks. Transformations were conducted, but these data could not be normalized. All four tasks had high ceiling effects. The square root transformation was conducted for the Pencil Tapping data and for the conduct subscale, but they still could not be normalized. Both measures still showed high kurtosis; these measures were thus precluded from the analyses. In the analyses that follow, the Huynh-Feldt correction was applied to some results due to violations of sphericity assumptions. Partial $\eta^{2}$ was used as a measure of effect size with using cutoffs for small effect at 0.02 , medium effect at 0.13 , and large effect at 0.26 (Cohen, 1988).

\section{Outcome Analyses}

The sample size limited the outcome analyses to the level of treatment condition, rather than the unit of randomization, and school characteristics could be confounded with child level variables. Hence, to address the extent to which the intervention impacted child performance on the behavioral measure of self-regulation (HTKS) and on teacher ratings of selfregulation and emotion regulation (SDQ and ERQ), RANCOVAs were first conducted to examine changes in variables over time as a function of the intervention. Preliminary results did not show an effect of parent education on any of the outcome measures. In the interest of making the models more parsimonious, parent education was dropped as a covariate from final analyses. Time 1 measures (e.g., HTKS at Time 1) were included in the model. The within-subject variable (time) had three levels, Time 1, Time 2, and Time 3. There were three independent variables in the model: treatment, time, and grade level. The focus of the RANCOVAs analyses was on identifying the interaction between treatment (TxFirst and TxSecond) and time, because this interaction was most relevant to the hypothesis of this study.

To supplement the results of the RANCOVAs and determine when significant changes in the outcome measures happened at specific time points, pairwise comparisons analyses were conducted for TxFirst and TxSecond of scores for all time points. The focus for TxFirst was on identifying significant differences in scores between Time 1 and Time 2 and between Time 1 and Time 3 (i.e., to see if the average scores on outcome measures were different across time points) and between Time 2 and Time 3 (i.e., to see if the average scores on outcome measures were sustained). For TxSecond, the focus was on identifying significant differences between scores for Time 1 and Time 3, and between Time 2 and Time 3 (i.e., to see if the average scores on outcome measures were changed from baseline). To correct for possible type I errors, pairwise comparisons with Bonferroni corrections (alpha was set to $0.05 / 3$ or 0.0167 ) were conducted for each treatment group between each possible pair of time points (T1 and $\mathrm{T} 2$, $\mathrm{T} 1$ and $\mathrm{T} 3$, and $\mathrm{T} 2$ and $\mathrm{T} 3$ ). The pairwise comparisons were used to see if there were significant improvements on outcome measures across time points. Descriptive statistics for each measure at each time point are presented in Table 2. For our

Table 2 Descriptive statistics for outcome variables on Children's Emotion Regulation Processes Questionnaire (ERQ), Head Toes Knees Shoulders (HTKS), and Strengths and Difficulties Questionnaire (SDQ) measures by treatment group and time point

\begin{tabular}{|c|c|c|c|c|c|c|c|c|c|c|c|c|}
\hline & \multicolumn{6}{|l|}{ TxFirst } & \multicolumn{6}{|l|}{ TxSecond } \\
\hline & \multicolumn{2}{|l|}{ Time 1} & \multicolumn{2}{|l|}{ Time 2} & \multicolumn{2}{|l|}{ Time 3} & \multicolumn{2}{|l|}{ Time 1} & \multicolumn{2}{|l|}{ Time 2} & \multicolumn{2}{|l|}{ Time 3} \\
\hline & $M$ & $S D$ & $M$ & $S D$ & $M$ & $S D$ & $M$ & $S D$ & $M$ & $S D$ & $M$ & $S D$ \\
\hline ERQ - Attention Focusing & 119.34 & 37.64 & $122.90^{\mathrm{b}}$ & 39.70 & $116.20^{\mathrm{b}}$ & 37.87 & 110.13 & 50.48 & 110.01 & 43.47 & 115.15 & 33.94 \\
\hline ERQ - Dominant Venting & 25.49 & 15.86 & 24.22 & 14.67 & 24.34 & 10.41 & 32.56 & 23.57 & 34.91 & 26.99 & 29.33 & 17.79 \\
\hline ERQ - Problem Focusing & $91.89^{\mathrm{c}}$ & 27.87 & $90.69^{\mathrm{b}}$ & 23.72 & $106.77^{\mathrm{b}, \mathrm{c}}$ & 22.54 & $94.41^{\mathrm{c}}$ & 32.48 & 101.44 & 33.04 & $105.40^{\mathrm{c}}$ & 22.79 \\
\hline ERQ - Submissive Venting & $39.68^{\mathrm{a}, \mathrm{c}}$ & 23.29 & $31.33^{\mathrm{a}}$ & 19.07 & $31.18^{\mathrm{c}}$ & 16.26 & $51.61^{\mathrm{c}}$ & 30.92 & $51.13^{\mathrm{b}}$ & 28.80 & $37.37^{\mathrm{b}, \mathrm{c}}$ & 21.73 \\
\hline HTKS & $23.42^{\mathrm{a}, \mathrm{c}}$ & 18.70 & $30.67^{\mathrm{a}}$ & 18.55 & $33.09^{c}$ & 19.59 & $21.42^{\mathrm{a}, \mathrm{c}}$ & 18.62 & $28.81^{\mathrm{a}, \mathrm{b}}$ & 20.78 & $36.58^{\mathrm{b}, \mathrm{c}}$ & 19.07 \\
\hline $\begin{array}{l}\text { SDQ - Emotion Regulation (Square } \\
\text { Root transformation) }\end{array}$ & .65 & .88 & $.63^{\mathrm{b}}$ & .83 & $.83^{\mathrm{b}}$ & .79 & 1.15 & .97 & 1.06 & .88 & .97 & .84 \\
\hline SDQ - Hyperactivity & 4.66 & 1.88 & $4.39^{\mathrm{b}}$ & 1.40 & $4.74^{\mathrm{b}}$ & 1.29 & 5.06 & 1.26 & 4.92 & 1.46 & 5.09 & 1.28 \\
\hline SDQ - Total Behavioral Difficulties & $12.58^{\mathrm{a}}$ & 3.25 & $11.87^{\mathrm{a}}$ & 2.36 & 12.82 & 2.67 & 13.61 & 3.08 & 13.20 & 3.76 & 13.00 & 2.87 \\
\hline
\end{tabular}

Significance of paired comparisons is noted

Values sharing the same superscript differ significant at $p<0.0167$

The intervention occurred between Time 1 and Time 2 for the TxFirst group, and between Time 2 and Time 3 for the TxSecond group 
Fig. 2 Mean submissive venting score at each time point for both treatment groups

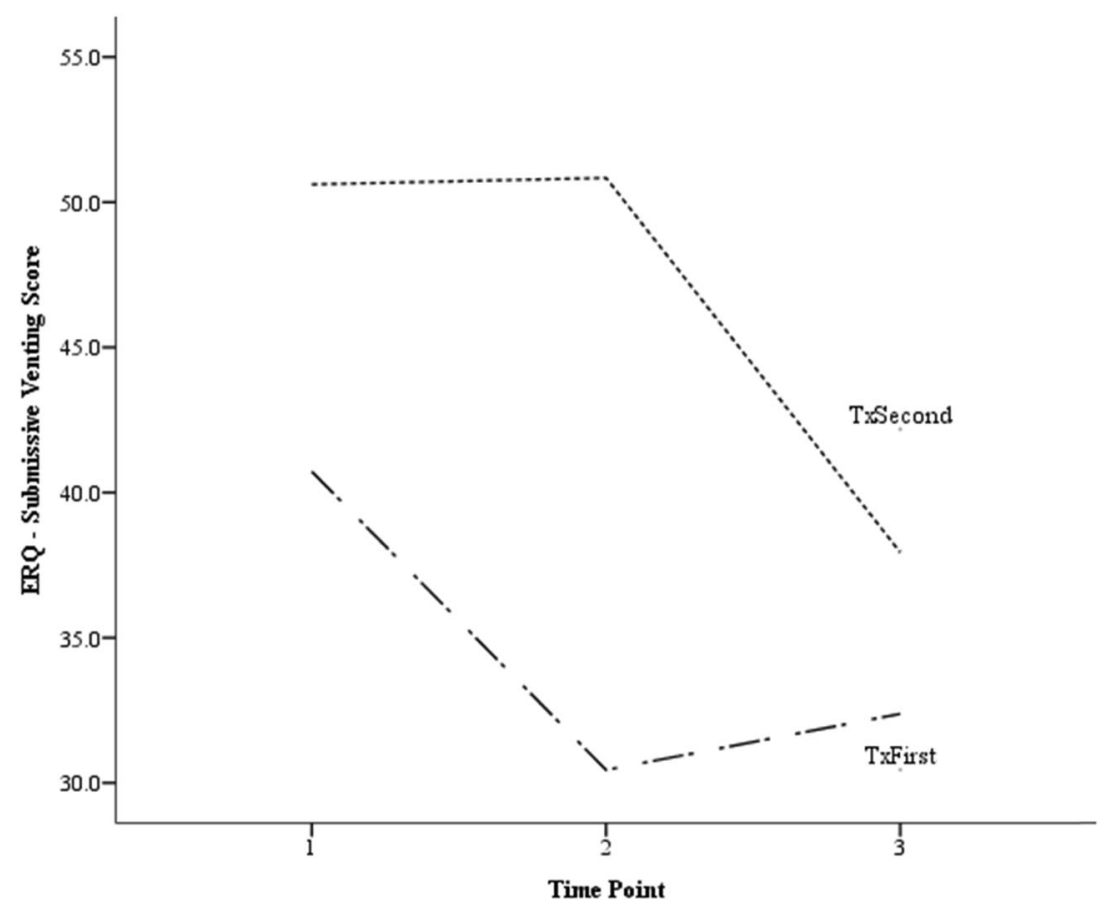

analyses, $p<0.05$ was interpreted as significant and $p<0.10$ was interpreted as marginally significant.

\section{Results}

In this study, we hypothesized that after the children received the intervention, they would demonstrate significant improvements on the task of self-regulation (HTKS) and on teacher ratings of self-regulation and emotion regulation (SDQ and ERQ). The results that follow demonstrate the extent to which the average scores on outcome measures were significantly different across time points.

\section{HTKS task}

For self-regulation assessed during the HTKS task, the RANCOVA did not show a significant interaction between time and treatment, although there was a marginally significant effect $F(2,297.546)=2.485, p=0.08$, and a small effect size (partial $\eta^{2}=0.016$ ). Paired comparisons were significant for TxFirst, between $\mathrm{T} 1$ and $\mathrm{T} 2, F(2,89)=$ 18.23, $p<0.001$ and between $\mathrm{T} 1$ and T3, $F(2,89)=27.02$, $p<0.001$, but not significant between T2 and T3, $F(2,89)$ $=2.51, p=0.117$. These findings suggest that students in TxFirst increased their HTKS scores immediately after receiving the intervention and maintained their gains after the intervention. Comparisons were significant for TxSecond, between T2 and T3, $F(2,63)=18.98, p<0.001$ and between $\mathrm{T} 1$ and $\mathrm{T} 3, F(2,63)=58.14, p<0.001$. This suggests that students in TxSecond increased their HTKS scores after receiving the intervention.

\section{Dominant venting-ERQ}

For dominant venting, the RANCOVA did not show a significant interaction between time and treatment $F(2$, $284)=0.877, p=0.417$, partial $\eta^{2}=0.006$. For dominant venting, paired comparisons were not significant for TxFirst, between $\mathrm{T} 1$ and $\mathrm{T} 2, F(2,89)=0.861, p=0.356$; between $\mathrm{T} 1$ and $\mathrm{T} 3, F(2,88)=0.047, p=0.830$; and between $\mathrm{T} 2$ and $\mathrm{T} 3, F(2,88)=0.924, p=0.339$. This suggests that students in TxFirst maintained equivalent dominant venting scores throughout the study. Comparisons were not significant for TxSecond, between T1 and T3, $F(2$, $56)=0.012, p=0.913$ and between $\mathrm{T} 2$ and $\mathrm{T} 3, F(2,56)=$ $0.984, p=0.326$. This suggests that students in TxSecond maintained equivalent dominant venting scores throughout the study.

\section{Submissive venting-ERQ}

For submissive venting, the RANCOVA revealed a significant interaction between treatment and time $F(1.985$, $297.805)=6.465, p=0.002$, partial $\eta^{2}=0.041$. The pairwise comparisons were significant for TxFirst, between T1 and $\mathrm{T} 2, F(2,89)=8.556, p=0.004$ and between $\mathrm{T} 1$ and T3, $F(2,89)=14.145, p<0.001$, but not between $\mathrm{T} 2$ and 
T3, $F(2,89)=0.004, p=0.948$. This suggests that students in TxFirst decreased their submissive venting scores immediately after receiving the intervention and sustained these scores at T3. For TxSecond, comparisons were significant between $\mathrm{T} 1$ and $\mathrm{T} 3, F(2,63)=10.093, p=0.002$ and between $\mathrm{T} 2$ and T3, $F(2,63)=18.148, p=0.001$. This suggests that students in TxSecond decreased their submissive venting scores immediately after receiving the intervention (see Fig. 2 for plots of scores for TxFirst and TxSecond).

\section{Attention focusing-ERQ}

For attention focusing, the RANCOVA did not show a significant interaction between time and treatment $F(1.935$, $290.254)=2.502, p=0.086$, partial $\eta^{2}=0.016$. For attention focusing, pairwise comparisons were not significant for TxFirst, between $\mathrm{T} 1$ and $\mathrm{T} 2, F(2,89)=1.713, p=0.194$ and between $\mathrm{T} 1$ and $\mathrm{T} 3, \mathrm{~F}(2,89)=0.749, p=0.389$ but were significant for between $\mathrm{T} 2$ and $\mathrm{T} 3, F(2,89)=5.726, p$ $=0.019$. This suggests that students in TxFirst maintained their attention focusing scores throughout the study, although their scores decreased between T2 and T3. Comparisons were not significant for TxSecond between $\mathrm{T} 1$ and T3, $F(2,63)=0.835, p=0.364$ and between $\mathrm{T} 2$ and T3, $F$ $(2,63)=1.153, p=0.287$. This suggests that students in TxSecond maintained equivalent attention focusing scores throughout the study.

\section{Problem-emotion focusing-ERQ}

For problem-emotion focusing, the RANCOVA did not show a significant interaction between time and treatment $F$ $(2,300)=1.779, \quad p=0.171$, partial $\eta^{2}=0.012$. For problem-emotion focusing, pairwise comparisons were not significant for TxFirst, between $\mathrm{T} 1$ and $\mathrm{T} 2, \mathrm{~F}(2,89)=$ $0.179, p=0.672$ but were significant for between $\mathrm{T} 1$ and $\mathrm{T} 3, F(2,89)=17.231, p<0.001$ and between $\mathrm{T} 2$ and T3, $F$ $(2,89)=21.772, p<0.001$. This suggests that students in TxFirst maintained problem focusing scores through T2 and increased their scores when measured at T3. For TxSecond, comparisons were not significant between $\mathrm{T} 2$ and T3, $F(2$, $63)=1.201, p=0.277$ but were significant between $\mathrm{T} 1$ and $\mathrm{T} 3, F(2,63)=9.290, p=0.003$. These findings suggest that students in TxSecond increased their problem focusing scores throughout the study.

\section{Hyperactivity-SDQ}

For hyperactivity, the RANCOVA did not show a significant interaction between time and treatment $F(1.899$, 284.902) $=0.367, \quad p=0.682$, partial $\eta^{2}=0.002$. For hyperactivity, pairwise comparisons were not significant for TxFirst between $\mathrm{T} 1$ and T2, $F(2,89)=2.068, p=0.154$ and between $\mathrm{T} 1$ and $\mathrm{T} 3, F(2,89)=0.197, p=0.658$. Comparisons were significant between $\mathrm{T} 2$ and $\mathrm{T} 3, F(2,89)$ $=7.306, p<0.01$. This suggests that students in TxFirst maintained equivalent hyperactivity scores after receiving the intervention, although hyperactivity scores increased at T3. Comparisons were not significant for TxSecond between $\mathrm{T} 1$ and $\mathrm{T} 3, F(2,63)=0.044, \mathrm{p}=0.835$ and between $\mathrm{T} 2$ and $\mathrm{T} 3, F(2,63)=0.922, p=0.340$. Overall, students in TxSecond maintained equivalent hyperactivity scores throughout the study.

\section{Total behavior difficulties-SDQ}

For total behavioral difficulties, the RANCOVA did not show a significant interaction between time and treatment $F$ $(1.858,278.704)=2.277, p=0.108$, partial $\eta^{2}=0.015$. For total behavioral difficulties, pairwise comparisons were significant for TxFirst, between $\mathrm{T} 1$ and $\mathrm{T} 2, F(2,89)=$ 5.953, $p=0.017$. Comparisons were not significant for TxFirst between $\mathrm{T} 1$ and T3, $F(2,89)=0.442, p=0.508$ but they were significant between $\mathrm{T} 2$ and $\mathrm{T} 3, F(2,89)=$ $11.116, p<0.01$. This suggests that students in TxFirst decreased their total behavioral difficulties scores immediately after receiving the intervention but did not maintain their gains throughout the study. Comparisons were not significant for TxSecond, between $\mathrm{T} 1$ and $\mathrm{T} 3, F(2,63)=$ $1.555, p=0.217$ and $\mathrm{T} 2$ and $\mathrm{T} 3, F(2,63)=0.327, p=$ 0.569. This finding suggests that students in TxSecond maintained equivalent total behavioral difficulties scores throughout the study.

\section{Emotion regulation-SDQ}

Data for the emotion regulation subscale were positively skewed and the square root transformation was taken for the RANCOVA. For emotion regulation, the RANCOVA did not show a significant interaction between time and treatment $F(1.043,294.39)=2.549, p=0.081$, partial $\eta^{2}=$ 0.017 . Pairwise comparisons were not significant for TxFirst, between $\mathrm{T} 1$ and $\mathrm{T} 2, F(2,89)=0.072, p=0.789$ and between $\mathrm{T} 1$ and $\mathrm{T} 3, F(2,89)=3.568, p=0.062$. Comparisons were significant between $\mathrm{T} 2$ and $\mathrm{T} 3, F(2,89)$ $=4.769, p=0.032$. This suggests that students in TxFirst maintained comparable emotion regulation scores from $\mathrm{T} 1$ to $\mathrm{T} 2$ but experienced an increase in scores between $\mathrm{T} 2$ and T3. For TxSecond, comparisons were not significant between $\mathrm{T} 1$ and $\mathrm{T} 3, F(2,63)=2.07, \mathrm{p}=0.219$ and between $\mathrm{T} 2$ and $\mathrm{T} 3, F(2,63)=1.54, p=0.395$. This suggests that students in TxSecond maintained equivalent emotion regulation scores throughout the study. 


\section{Discussion}

The purpose of our study was to investigate the effects of an implementation of a yoga intervention that targeted self-regulation and emotion regulation in prekindergarten and kindergarten classrooms. An advantage of this study was that the teachers implemented the intervention with a manualized set of videos, thereby enhancing the ecological validity of the findings and the FOI, with respect to children consistently receiving yoga practices (Maynard et al., 2017). We also addressed the need to have teachers as primary implementers, while not requiring a substantial amount of teachers' time and providing teachers with the intervention supports for implementation (Mendelson et al., 2013). The treatment effects were observed after a short intervention period, which corroborates findings in prior yoga intervention research involving young children (Flook et al., 2015; Poehlmann-Tynan et al., 2016; Razza et al., 2013; Zelazo et al., 2018).

Overall, the results provided some support for our hypothesis, in that the yoga intervention was associated with predicted improvements several measures of selfregulation. On the behavioral measure of self-regulation (HTKS), an intervention effect was observed for both TxFirst (improving from $\mathrm{T} 1$ to $\mathrm{T} 2$ and sustained at $\mathrm{T} 3$ ) and TxSecond (improving from T2 to T3). Evidence for intervention effects were also observed on several teacher ratings. On the measure of submissive venting, for example, there was an intervention effect for TxFirst and TxSecond (indicating a decline in crying from anger or frustration), and this effect was sustained for children in TxFirst (see Fig. 2). Similarly, on ratings of total behavior problems, there was an intervention effect for TxFirst (indicating a decline in problem behaviors) that was not sustained, although there was no effect for TxSecond. The other teacher ratings did not show significant intervention effects.

The intervention included breath regulation, postures, and developing awareness of sensations - all of which could have contributed to children's abilities to effectively identify, regulate, and manage their emotions. Intervention effects were not found on measures gauging metacognitive processes, such as attention focusing, but were more evident on these emotion-based subscales (e.g., submissive venting and problem-emotion focused coping). This suggests that yoga helped children regulate emotions, perhaps by showing children how to become aware of and manage their bodily sensations (Gard et al., 2014; Moreno, 2017).

For other measures, and consistent with prior research, a sleeper effect could have been at play, in that these skills take time for generalization to the context of self- regulation assessments (Zelazo et al., 2018). This might help to explain the improvement in problem-emotion focused coping at $\mathrm{T} 3$ for both intervention groups, despite the different times when the intervention occurred, and also the improvement in teacher ratings of emotion regulation from $\mathrm{T} 2$ to $\mathrm{T} 3 \mathrm{in}$ the TxFirst group. A sleeper effect might be likely given that children were new to the yoga practices. Hence, children may have needed more time to generalize the skills learned from the intervention to the context of the direct assessment and teacher ratings of outcome measures. For the remaining measures, there was little evidence for an intervention effect. The mean scores for dominant venting suggest that the behaviors indexed by this scale (e.g., hitting, yelling) were fairly rare and were difficult for teachers to reliably rate; the same might be true of teacher ratings of hyperactivity. It was also suprising that students in TxFirst showed an increase in hyperactivity at $\mathrm{T} 3$. This may have been a result of the intervention needing to be longer in order for the longer-term effects of a decrease in hyperactivity to show. It is possible that the post-intervention period provided insufficient time to detect effects on these measures. Futhermore, it may be the case that throughout the course of this intervention, the teachers grew more aware of their students' behaviors on the hyperactivity subscale. This could also explain the increase in hyperactivity at $\mathrm{T} 3$ for students in TxFirst.

\section{Limitations and Future Research}

There are a number of limitations to this study. The sample size limited the analyses to the level of treatment condition rather than the unit of randomization (Maynard et al., 2017). A larger sample was needed to conduct hierarchical linear modeling and use schools as a second level variable. Consistent with prior studies, school characteristics could be confounded with child level variables (Flook et al., 2015; Poehlmann-Tynan et al., 2016). Additionally, some variables were omitted from analysis because of non-normal data distributions arising from a high ceiling effect due to participants performing higher than expected for the Pencil Tapping task, conduct SDQ subscale, and the Toy Wrap and Toy Touch tasks. Although we successfully transformed the emotion regulation SDQ subscale scores to fit the assumptions required for RANCOVA analyses, the other variables were dropped from the study. This affects our interpretation of the effects of the intervention on selfregulation as there were fewer outcomes to analyze. This study also lacked a long-term follow-up assessment with which to corroborate the nearer-term effects observed in these results, even though two follow-up assessments is unusual in this literature. Intervention duration did not 
allow for detecting sustained effects. In comparison to students in TxSecond, students in TxFirst generally demonstrated greater effects on measures; thus, the manualized intervention did not produce identical results across both groups. Future research is needed on measuring the differences in intervention efficacy based on child characteristics (e.g., temperament) and teacher characteristics. On a related note, intervention effects could have been attributed to maturation, rather than to the intervention itself (Shadish et al., 2002). Moreover, intervention effects were not documented in other contexts, such as the home, as parent ratings of child behavior were not collected. This is a limitation, although the focus of this study was to see if the intervention would impact self-regulation and emotion regulation outcomes in the classroom setting. As such, teacher ratings were only collected. In addition, this study required $10 \mathrm{~h}$ of training. This time commitment might have dissuaded other teachers from participating in the study.

Future researchers interested in a broader dissemination and implementation of yoga interventions may consider alternative approaches to teacher training. For example, the $10 \mathrm{~h}$ of in-person teacher training could be conducted in a combination of a shorter in-person workshop, followed up by asynchronous online modules for teachers to complete. Additionally, teachers were not blind to the treatment condition, and this could have resulted in biased scores on the SDQ and ERQ. Practice effects could also have been at play with behavioral measures, such as the HTKS task, as these tasks can be influenced by practice effects. Nevertheless, McClelland et al. (2014) study, a study examining the psychometric properties of the HTKS task, reported that there were significant improvements on HTKS scores between spring and fall for Pre-Kindergarten and Kindergarten students, along with other measures of executive functioning. Practice effects could have been at play in that study, as well as in our study, as executive functions already improve during this developmental period. Furthermore, participants volunteered to participate in this study and selection bias could be at play (Shadish et al., 2002).

Despite these limitations, this study is one of the few to have involved teachers as the primary implementers, as roughly $70 \%$ of yoga and mindfulness interventions have been implemented by professionals external to the school (Maynard et al., 2017). Such intervention models might not be sustainable nor preferred by teachers in the long-term (Dariotis et al., 2017; Mendelson et al., 2013). Consistent with the call in prior research for providing teachers with the resources to faclitate yoga practices, we ensured teachers had the training and video supports to implement brief yoga sessions into their classrooms (Dariotis et al., 2017; Mendelson et al., 2013). A potential strength of the video supports we provided was that teachers would ideally be able to integrate these practices into their classrooms beyond the eight-week intervention and throughout the entire year. However, follow-up studies would be needed to assess the extent to which the effects of a year-long implementation would be sustained. Overall, our findings demonstrated that a manualized, teacher-guided eight-week yoga intervention can result in some measurable improvements in pre-kindergarten and kindergarten children's selfregulation, but additional research is needed to determine how generalizable these effects are. Cultivating such selfregulatory skills early in life may improve children's academic success and emotional well-being and thus enhance their overall developmental paths. Specifically, the sample of children in this study included a high proportion from families in economic difficulty, adding to the evidence for the potential benefits of yoga instruction for children under stress (Poehlmann-Tynan et al., 2016; Razza et al., 2020). Furthermore, as children transition back to school settings after a year or more of pandemic-related sheltering at home, yoga practices may become even more helpful in enabling children to sustain emotional self-control in the context of group learning and enhanced peer interaction.

Acknowledgements We thank the teachers, school staff, students, yoga studio, and research assistants for their participation and commitment to this study. Thank you to The Mind \& Life Institute (Grant ID Number: A-13146632) for their financial support of this line of inquiry.

Author Contributions R.N.R.: designed and executed the study, assisted with data analyses, and wrote the paper. S.R.: ran the analyses and assisted with writing the paper. R.A.T.: assisted with data analyses and assisted with writing the paper. E.J.S.: assisted in the editing of the final manuscript. K.A.S.R.: assisted in the editing of the final manuscript.

\section{Compliance with Ethical Standards}

Conflict of Interest In accordance with Springer's policy and our ethical obligations as researchers, we are reporting that we do not have a financial and/or business interests in a company that may be affected by the research reported in the enclosed paper. Any views, findings, conclusions, or recommendations expressed in this article do not necessarily reflect those of the Mind \& Life Institute.

Ethical Approval The protocols used in this research study were approved by the ethics committee at the University of California, Davis in Davis, California.

Informed Consent Informed consent was obtained from all participants included in the study.

Publisher's note Springer Nature remains neutral with regard to jurisdictional claims in published maps and institutional affiliations. 


\section{Appendix}

\section{Sample Lesson}

Seated Pretzel: Crossing the Midline

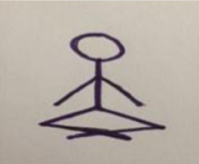

Movement 1: Seated Hug Knees to

Chest Pose

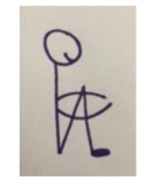

Movement 2: All Fours: Cat and Cow Stretches

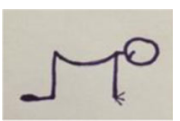

Movement 3: Cobra Pose

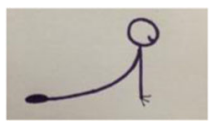

Movement 4: Child's Pose

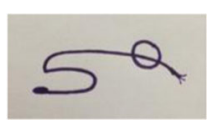

Relaxation Pose

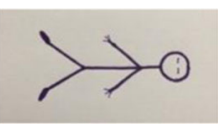

\section{References}

Barkley, R. A. (2001). The executive functions and self-regulation: an evolutionary neuropsychological perspective. Neuropsychology Review, 11(1), 1-29. https://doi.org/10.1023/a:1009085417776.

Bernzweig, J., Eisenberg, N., \& Fabes, R. A. (1993). Children's coping with self- and other-relevant contexts. Journal of Experimental Child Psychology, 55, 208-226. https://doi.org/10. 1006/jecp.1993.1012.

Blair, C., \& Razza, R. P. (2007). Relating effortful control, executive function, and false belief understanding to emerging math and literacy ability in kindergarten. Child development, 78(2), 647-663. https://doi.org/10.1111/j.1467-8624.2007.01019.x.

Blair, C., \& Diamond, A. (2008). Biological processes in prevention and intervention: the promotion of self-regulation as a means of preventing school failure. Development and Psychopathology, 20, 899-911. https://doi.org/10.1017/s0954579408000436.
Bourdon, K. H., Goodman, R., Rae, D. S., Simpson, G., \& Koretz, D. S. (2005). The Strengths and Difficulties Questionnaire: US normative data and psychometric properties. Journal of the American Academy of Child \& Adolescent Psychiatry, 44(6), 557-564. https://doi.org/10.1097/01.chi.0000159157.57075.c8.

Cohen, J. (1988). Statistical power analysis for the behavioral sciences. 2nd ed. New York, NY: Academic Press.

Collins, B. (2015). Sensory yoga for kids: therpeautic movement for children of all abilities. Arlington, TX: Sensory World, LLC.

Dariotis, J. K., Mirabal-Beltran, R., Cluxton-Keller, F., Feagans Gould, L., Greenberg, M. T., \& Mendelson, T. (2017). A qualitative exploration of implementation factors in a school-based mindfulness and yoga program: Lessons learned from students and teachers. Psychology in the Schools, 54(1), 53-69. https://doi. org/10.1002/pits.21979.

Davidson, R., Dunne, J., Eccles, J. S., Engle, A., Greenberg, M., Jennings, P., \& Roeser, R. W. (2012). Mindful practices and mental training: Prospects for American education. Child Development Perspectives, 6(2), 146-153. https://doi.org/10. 1111/j.1750-8606.2012.00240.x.

Diamond, A. (2013). Executive functions. Annual review of psychology, 64, 135-168. https://doi.org/10.1146/annurev-psych113011-143750.

Diamond, A., \& Lee, K. (2011). Interventions shown to aid executive function development in children 4 to 12 years old. Science, 333 (6045), 959-964. https://doi.org/10.1126/science.1204529.

Feagans Gould, L., Dariotis, J. K., Greenberg, M. T., \& Mendelson, T. (2015). Assessing fidelity of implementation (FOI) for schoolbased mindfulness and yoga interventions: a systematic review. Mindfulness, 7(1), 5-33. https://doi.org/10.1007/s12671-0150395-6.

Flook, L., Goldberg, S. B., Pinger, L., \& Davidson, R. J. (2015). Promoting prosocial behavior and self-regulatory skills in preschool children through a mindfulness- based kindness curriculum. Developmental psychology, 51(1), 44 https://doi.org/10. 1037/a0038256.

Gard, T., Noggle, J. J., Park, C. L., Vago, D. R., \& Wilson, A. (2014). Potential self-regulatory mechanisms of yoga for psychological health. Frontiers in Human Neuroscience, 8, 770 https://doi.org/ 10.3389/fnhum.2014.00770.

Greenberg, M. T., Weissberg, R. P., O’Brien, M. U., Zins, J. E., Fredericks, L., Resnik, H., \& Elias, M. J. (2003). Enhancing schoolbased prevention and youth development through coordinated social, emotional, and academic learning. American psychologist, 58(6-7), 466 https://doi.org/10.1037/003-066x.58.6-7.466.

Hallgren, K. A. (2012). Computing inter-rater reliability for observational data: An overview and tutorial. Tutorials in Quantitative Methods for Psychology, 8(1), 23-34. https://doi.org/10.20982/ tqmp.08.1.p023.

Hart, J. (2020). Pandemic Drives Increase in Mind-Body Therapy Use: Implications for the Future. Alternative and Complementary Therapies, 26(6), 243-245. https://doi.org/10.1089/act.2020. 29298.jha.

Hedges, L. V., \& Rhoads, C. (2010). Statistical power analysis in education research. NCSER 2010-3006. National Center for Special Education Research. https://doi.org/10.1016/b978-0-08044894-7.01356-7

Jarraya, S., Wagner, M. O., Jarraya, M., \& Engel, F. A. (2019). 12 weeks of Kindergarten-based yoga practice increases visual attention, visual-motor precision and decreases behavior of inattention and hyperactivity in 5-year-old children. Frontiers in psychology, 10, 796 https://doi.org/10.3389/fpsyg.2019.00796.

Lawlor, M. S. (2016). Mindfulness and social emotional learning (SEL): A conceptual framework. In K. A. Schonert-Reichl \& R. W. Roeser (Eds.), Handbook of mindfulness in education (pp. 65-80). New York, NY: Springer. 
Majumdar, V., Nagarathna, R., \& Nagendra, H. (2020). A perspective on yoga as a preventive strategy for coronavirus disease 2019. International Journal of Yoga, 13(2), 89 https://doi.org/10.4103/ ijoy.ijoy_22_20.

Maynard, B. R., Solis, M. R., Miller, V. L., \& Brendel, K. E. (2017). Mindfulness-based interventions for improving cognition, academic achievement, behavior, and socioemotional functioning of primary and secondary school students. Campbell Systematic Reviews, 5, 1-147. https://doi.org/10.4073/csr.2017.5.

McClelland, M. M., Acock, A. C., Piccinin, A., Rhea, S. A., \& Stallings, M. C. (2013). Relations between preschool attention spanpersistence and age 25 educational outcomes. Early Childhood Research Quarterly, 28(2), 314-324. https://doi.org/10.1016/j. ecresq.2012.07.008.

McClelland, M. M., Cameron, C. E., Duncan, R., Bowles, R. P., Acock, A. C., Miao, A., \& Pratt, M. E. (2014). Predictors of early growth in academic achievement: the head-toes-knees-shoulders task. Frontiers in psychology, 5, 599 https://doi.org/10.3389/ fpsyg.2014.00599.

McHugh, M. L. (2012). Interrater reliability: the kappa statistic. Biochemia medica, 22(3), 276-282. https://doi.org/10.11613/bm.2012.031.

Mendelson, T., Greenberg, M. T., Dariotis, J. K., Gould, L. F., Rhoades, B. L., \& Leaf, P. J. (2010). Feasibility and preliminary outcomes of a school-based mindfulness intervention for urban youth. Journal of Abnormal Child Psychology, 38(7), 985-994. https://doi.org/10.1007/s10802-010-9418-x.

Mendelson, T. K., Dariotis, J., Feagans Gould, L., Sr Smith, A., Smith, A., Gonzalez, A., \& Greenberg, T. M. (2013). Implementing mindfulness and yoga in urban schools: a community-academic partnership. Journal of Children's Services, 8(4), 276-291. https://doi.org/10.1108/jcs-07-2013-0024.

Meyer, S., Raikes, H. A., Virmani, E. A., Waters, S., \& Thompson, R. A. (2014). Parent emotion representations and the socialization of emotion regulation in the family. International Journal of Behavioral Development, 38(2), 164-173. https://doi.org/10. 4324/9780203080719.

Moreno, A. J. (2017). A theoretically and ethically grounded approach to mindfulness practices in the primary grades. Childhood Education, 93(2), 100-108. https://doi.org/10.1080/00094056.2017.1300487.

Poehlmann-Tynan, J., Vigna, A. B., Weymouth, L. A., Gerstein, E. D., Burnson, C., Zabransky, M., \& Zahn-Waxler, C. (2016). A pilot study of contemplative practices with economically disadvantaged preschoolers: children's empathic and self-regulatory behaviors. Mindfulness, 7(1), 46-58. https://doi.org/10.1007/ s12671-015-0426-3.

Ponitz, C. C., McClelland, M. M., Matthews, J. S., \& Morrison, F. J. (2009). A structured observation of behavioral self-regulation and its contribution to kindergarten outcomes. Developmental psychology, 45(3), 605 https://doi.org/10.1037/a0015365.

Razza, R. A., Bergen-Cico, D., \& Raymond, K. (2013). Enhancing preschoolers' self- regulation via mindful yoga. Journal of Child and Family Studies, 24(2), 372-385. https://doi.org/10.1007/ s10826-013-9847-6.

Razza, R. A., Linsner, R. U., Bergen-Cico, D., Carlson, E., \& Reid, S. (2020). The feasibility and effectiveness of mindful yoga for preschoolers exposed to high levels of trauma. Journal of Child and Family Studies, 29(1), 82-93. https://doi.org/10.1007/ s10826-019-01582-7.

Rimm-Kaufman, S. E., Pianta, R. C., \& Cox, M. J. (2000). Teachers' judgments of problems in the transition to kindergarten. Early Childhood Research Quarterly, 15(2), 147-166. https://doi.org/ 10.1016/s0885-2006(00)00049-1.

Roeser, R. W., \& Peck, S. C. (2009). An education in awareness: Self, motivation, and self-regulated learning in contemplative perspective. Educational Psychologist, 44(2), 119-136. https:// doi.org/10.1080/00461520902832376.

Schmalzl, L., Crane-Godreau, M. A., \& Payne, P. (2014). Movementbased embodied mindful practices: definitions and paradigms. Frontiers in Human Neuroscience, 8. https://doi.org/10.3389/ fnhum.2014.00205

Schonert-Reichl, K. A. (2019). Advancements in the Landscape of Social and Emotional Learning and Emerging Topics on the Horizon. Educational Psychologist, 54(3), 222-232. https://doi. org/10.1080/00461520.2019.1633925.

Schonert-Reichl, K. A., Oberie, E., Stewart Lawlor, M., Abbott, D., Thomson, K., Oberlander, T. F., \& Diamond, A. (2015). Enhancing cognitive and social-emotional development through a simple-to-administer mindfulness-based school program for elementary school children: a randomized controlled trial. Developmental Psychology, 51(1), 52-66. https://doi.org/10.1037/a 0038454.

Semple, R. J., Droutman, V., \& Reid, B. A. (2017). Mindfulness goes to school: Things learned (so far) from research and real-world experiences. Psychology in the Schools, 54(1), 29-52. https://doi. org/10.1002/pits.21981.

Shadish, W. R., Cook, T. D., \& Campbell, D. T. (2002). Experimental and quasi-experimental designs for generalized causal inference. Boston: Houghton Mifflin.

Shapiro, S. L., Carlson, L. E., Astin, J. A., \& Freedman, B. (2006). Mechanisms of mindfulness. Journal of Clinical Psychology, 62 (3), 373-386. https://doi.org/10.1002/jclp.20237.

Smith-Donald, R., Raver, C. C., Hayes, T., \& Richardson, B. (2007). Preliminary construct and concurrent validity of the Preschool Self-regulation Assessment (PSRA) for field-based research. Early Childhood Research Quarterly, 22(2), 173-187. https://doi. org/10.1016/j.ecresq.2007.01.002.

Stone, L. L., Otten, R., Engels, R. C. M. E., Vermulst, Ad A., \& Janssens, Jan M. A. M. (2010). Psychometric properties of the parent and teacher versions of the strengths and difficulties questionnaire for 4- to 12-year-olds: A review. Clinical Child and Family Psychology Review, 13(3), 254-274.

Stuart, E. A., \& Rubin, D. B. (2008). Best practices in quasiexperimental designs. Best practices in quantitative methods, $155-176$.

Von Suchodoletz, A., Trommsdorff, G., Heikamp, T., Wieber, F., \& Gollwitzer, P. M. (2009). Transition to school: the role of kindergarten children's behavior regulation. Learning and Individual Differences, 19(4), 561-566. https://doi.org/10.1016/j.lindif. 2009.07.006.

Thompson, R. A. (1994). Emotion regulation: a theme in search of definition. Monographs of the society for research in child development, 59(2-3), 25-52. https://doi.org/10.2307/1166137.

Thompson, R. A. (2019). Emotion dysregulation: a theme in search of definition. Development and Psychopathology, 31(3), 805-815. https://doi.org/10.1017/s0954579419000282.

Wolff, K., \& Stapp, A. (2019). Investigating early childhood teachers' perceptions of a preschool yoga program. SAGE Open, 9(1), 2158244018821758 https://doi.org/10.1177/2158244018821758.

Zelazo, P. D., \& Lyons, K. E. (2012). The potential benefits of mindfulness training in early childhood: a developmental social cognitive neuroscience perspective. Child Development Perspectives, 6, 154-160. https://doi.org/10.1111/j.1750-8606.2012. 00241.x.

Zelazo, P. D., Forston, J. L., Masten, A. S., \& Carlson, S. M. (2018). Mindfulness plus reflection training: effects on executive function in early childhood. Frontiers in Psychology, 9, 208 https://doi. org/10.3389/fpsyg.2018.00208. 